\title{
Análise comparativa da síntese de hidroxiapatita via estado sólido
}

\author{
Comparative analysis of solid state \\ hydroxyapatite synthesis
}

\author{
Jailton Romão Viana ${ }^{1}$, Ana Angélica Mathias Macêdo ${ }^{1,2}$, \\ Adenilson Oliveira dos Santos ${ }^{1}$, Pedro de Freitas Façanha Filho', \\ Manuel Pedro Fernandes Graça ${ }^{3}$, Manuel Almeida Valente ${ }^{3}$, \\ Cleber Cândido da Silva ${ }^{1}$
}

\author{
${ }^{1}$ Coordenação de Pós-Graduação em Ciência dos Materiais, Centro de Ciências Sociais, Saúde e Tecnologia/Campus \\ Imperatriz, Universidade Federal do Maranhão, Imperatriz, MA, Brasil. \\ e-mail: jailton.viana@ufma.br, adenilson1@gmail.com, freitasfacanha@gmail.com, cleber.silva@ufma.br \\ ${ }^{2}$ Instituto Federal de Educação, Ciência e Tecnologia do Maranhão, Imperatriz, MA, Brasil. \\ e-mail: anaangellica@yahoo.com.br \\ ${ }^{3}$ Departamento de Física, I3N, Universidade de Aveiro, CEP: 3810-193, Aveiro, Aveiro, Portugal. \\ e-mail:mpfg@ua.pt,mav@ua.pt
}

\begin{abstract}
RESUMO
A hidroxiapatita é um fosfato de cálcio considerada como biomaterial utilizada em restaurações ósseas, uma vez que apresenta características semelhantes à fase mineral do tecido ósseo. Por possuir diversos métodos de obtenção, a síntese do pó de hidroxiapatita tem estimulado interesse de pesquisas acadêmicas e indústrias, pois as condições de síntese e dos reagentes influenciam na estrutura, morfologia e aplicação do composto final desejado. Este trabalho propende realizar um estudo comparativo sobre síntese do pó de hidroxiapatita utilizando reagentes iguais, por dois métodos diferentes: mecanoquímica e calcinação. A síntese da hidroxiapatita foi realizada pela reação de estado sólido utilizando como precursores, o hidróxido de cálcio e o fosfato monoácido de cálcio em quantidades estequiométricas. As caracterizações foram realizadas por difração de raios X, espectroscopia Raman, microscopia eletrônica de varredura, espectroscopia de energia dispersiva, densidade, porosidade e microdureza Vickers. Os difratogramas de raios X apresentaram única fase para o método mecanoquímico e mais de uma fase para o método de calcinação, as fases foram confirmadas após refinamento pelo método de Rietveld e o tamanho médio do cristalito foi calculado pela equação de Scherrer. A espectroscopia Raman evidenciou bandas dos modos vibracionais da hidroxiapatita. As micrografias caracterizaram as diferenças morfológicas ocorridas nas amostras devido a diferentes métodos de síntese e a espectroscopia de energia dispersiva indicou as razões cálcio/fósforo das amostras, classificando-a como cerâmica à base de fosfato de cálcio. Medidas de densidades e porosidades das amostras foram realizadas, utilizando o método do picnômetro, baseado no princípio de Arquimedes. Os valores encontrados foram satisfatórios, pois estão próximos a valores teóricos encontrados na literatura. Medidas de microdureza Vickers foram obtidas nas amostras, com o intuito de averiguar suas propriedades mecânicas. Os métodos empregados se mostraram eficazes na produção de pós da hidroxiapatita.
\end{abstract}

Palavras-chave: hidroxiapatita, mecanoquímica, calcinação.

ABSTRACT
Hydroxyapatite is a calcium phosphate considered a biomaterial used in bone restorations, since it has char-
acteristics similar to the mineral phase of bone tissue. Due to its many methods of production, the synthesis
of hydroxyapatite powder has stimulated interest of academic research and industries, as the conditions of
synthesis and reagents influence the structure, morphology and application of the desired final compound.
This work aims to perform a comparative study on hydroxyapatite powder synthesis using equal reagents by
two different methods: mechanochemical and calcination. The synthesis of hydroxyapatite was performed
by solid state reaction using as precursors, calcium hydroxide and calcium monoacid phosphate in stoichio-
metric quantities. The characterizations were performed by X-ray diffraction, Raman spectroscopy, scanning 
electron microscopy, dispersive energy spectroscopy, density, porosity and Vickers microhardness. X-ray diffractograms showed a single phase for the mechanochemical method and more than one phase for the calcination method, the phases were confirmed after refinement by the Rietveld method and the average crystallite size was calculated by the Scherrer equation. Raman spectroscopy showed bands of the vibrational modes of hydroxyapatite. The micrographs characterized the morphological differences in the samples due to different synthesis methods and the dispersive energy spectroscopy indicated the calcium/phosphorus ratios of the samples, classifying it as calcium phosphate-based ceramic. Measurements of sample densities and porosities were performed using the pycnometer method based on the Archimedes principle. The values found were satisfactory, since they are close to theoretical values found in the literature. Vickers microhardness measurements were obtained in the samples, in order to verify their mechanical properties. The methods employed proved to be effective in the production of hydroxyapatite powders.

Keywords: hydroxyapatite, mechanochemical, calcination

\section{INTRODUÇÃO}

As aplicações de cerâmicas como biomaterial se destacam em áreas médicas e odontológicas, sendo empregada na substituição de juntas de quadris e de fêmur, dentes, vértebras e em reparo de ossículos do ouvido interno [1]. Sua importância se dá pelo fato de que as cerâmicas à base de fosfato de cálcio possuem composição análoga ao da matriz óssea e apresentam características como: biocompatibilidade, bioatividade, osteointegração, ausência em reações e/ou respostas inflamatória, reabsorbilidade gradual, tempos de pega e endurecimento controlável, com perfeita adesão ao tecido duro, condutor e estimulador na formação de tecido ósseo (osteocondução) [2, 3]. Contudo as propriedades mecânicas não são boas, pois apresentam baixa resistência à fadiga e tenacidade, limitando sua utilização em regiões de pouco esforço mecânico. Com o intuito de melhorar tais propriedades, diversos estudos vêm sendo realizado para reforçar microestruturalmente a matriz da hidroxiapatita a partir da formação de compósitos, destacando-se: alumina, mulita, titânio, zircônia e biovidros [4,5].

Em meio fisiológico, a hidroxiapatita pode atuar como revestimento de implantes ortopédicos, dentários e como reserva de cálcio e fósforo, pois há facilidade de substituições de ânions e cátions, que favorecem a liberação e/ou armazenamento de íons nos líquidos corporais, além de acelerar o crescimento ósseo ao redor do implante [6]. A hidroxiapatita (natural ou sintética) apresenta porosidade, tendo notável capacidade de adsorção de moléculas, como: proteínas, enzimas e aminoácidos, possibilitando a incorporação de fármacos para carreamento e liberação de drogas [7]. Sua porosidade auxilia tanto na redução de peso, como na capacidade de fornecer cálcio rapidamente para apoiar processos histológicos, favorecendo o desenvolvimento de ligações interfaciais entre a HAP e o tecido vivo [8]. Outra possível aplicação é na área ambiental, podendo operar como co-catalisador na decomposição de compostos orgânicos, auxiliando no controle de poluição ambiental devido sua alta capacidade de remover metais pesados da água e de solos contaminados [9].

A hidroxiapatita (HAP) é uma das cerâmicas mais estáveis e menos solúveis entre os fosfatos de cálcio, com fórmula molecular $\mathrm{Ca}_{10}\left(\mathrm{PO}_{4}\right)_{6}(\mathrm{OH})_{2}$. Possui estrutura cristalina hexagonal pertencente ao grupo

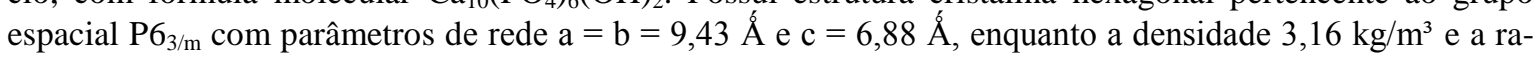
zão $\mathrm{Ca} / \mathrm{P}=1,67$, podendo ser estendida a valores próximos entre 1,5 e 2,0 [3,10]. A célula unitária (Figura 1) é constituída por 10 íons de cálcio $\left(\mathrm{Ca}^{2+}\right), 6$ grupos fosfatos $\left(\mathrm{PO}_{4}{ }^{3-}\right)$ e 2 íons de hidroxila $\left(\mathrm{OH}^{-}\right)$, havendo dois sítios de íons cálcio não equivalentes, o sítio I (CaI) e sítio II (CaII) [11,12]. Na Figura 1 , mostra os íons de cálcio do sítio I organizados em colunas paralelas, enquanto os íons de cálcio do sítio II formam a estrutura hexagonal.

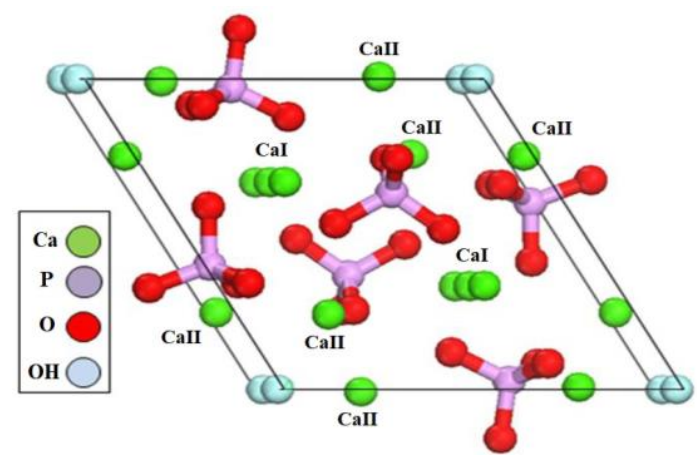

Figura 1: Célula unitária da hidroxiapatita [13]. 
Estudos sobre os métodos de síntese da hidroxiapatita vêm sendo muito explorados por pesquisadores, procurando-se o aprimoramento na produção de materiais de baixo custo e com maior resistência mecânica $[14,15]$. Sua produção a baixas temperaturas tem baixa cristalinidade, obtendo um material mais frágil. Quando preparada a altas temperaturas, apresenta boa cristalinidade, conseguindo-se produzir um material mais resistente e de fácil manipulação [16].

Como consequência, diversas investigações têm sido realizadas no que se refere aos métodos de síntese da hidroxiapatita, classificando-os em: úmido e seco. No processo úmido, as reações baseiam-se em soluções fazendo uso de um solvente e o subproduto é aquoso. São reações químicas moldáveis, todavia exigem condições controladas, na qual os parâmetros de reação devem ser ajustados, resultando em morfologia e tamanho médio da partícula homogêneos, diminuindo a contaminação do material, porém com fraca reprodutibilidade, redução na cristalinidade e alto custo, sendo viável para análises laboratoriais. Enquanto, o método seco não necessita de solvente, não é influenciado por parâmetros e condições controladas, favorecendo a alta reprodutibilidade e baixo custo, contudo pode aumentar o risco de contaminação, apresentar morfologia e tamanho médio das partículas heterogêneas, favorável para produção em escala industrial [17-19].

Dentre as várias possibilidades de síntese da hidroxiapatita no método seco, está a moagem mecânica de alta energia reativa, denominada mecanoquímica. A moagem mecânica de alta energia envolve a preparação de pós a partir de repetidos ciclos de deformação, re-soldagem à frio e fragmentação das partículas em um moinho de bolas de alta energia, capaz de sintetizar uma variedade de materiais metaestáveis e com melhor conformabilidade, sendo que a mecanoquímica é um caso particular da moagem mecânica na qual se refere a um processo de reações químicas e transformações de fase ocorrem devido à aplicação de energia mecânica, relacionado com as colisões entre as esferas - pó - esferas e esferas - pó - parede do recipiente $[20,21]$.

O método mecanoquímico é utilizado para a fabricação de uma gama de materiais avançados, metálicos e não-metálicos, na qual prepara material nanoestruturados cristalinos e/ou amorfos. Nas últimas décadas, tem sido empregado para as sínteses de materiais em pó, pois encurta o tempo de reação, reduz altas temperaturas e possibilita obtenção de materiais com propriedades específicas. [4, 21, 22]. Apesar das vantagens, a técnica oferece algumas desvantagens, tais como: escassa informação científica, pouca aplicação industrial e a contaminação do pó devido às condições de moagem, como: meio, recipiente, tempo e intensidade [20]

Outro método a seco em que a síntese da hidroxiapatita pode ser realizada é a calcinação, técnica que possui procedimento simples e robusto, remove impurezas indesejadas e pode acarretar melhoras na condutividade elétrica. A temperatura de calcinação leva o material sintetizado uma formação de estrutura bem cristalina, mas por outro lado, diminui a solubilidade e o pó pode exibir heterogeneidade em sua composição de fase, devido a pequenos coeficientes de difusão de íons dentro da fase sólida durante a reação de decomposição e difusão sólido-sólido do material. Esse método pode utilizar precursores que contenham Ca e fosfato ou sal de CaP previamente preparados [8, 11, 23].

Tal designação nominal "calcinação" refere-se a procedimentos já utilizados por civilizações antigas, quando transformavam calcário em cal ou óxido de cálcio, durante a queima do material [24]. Neste trabalho, apresentar-se-ão resultados comparativos da hidroxiapatita produzidas por reação de estado sólido de duas formas: mecanoquímica e calcinação, fazendo uso dos mesmos percussores.

\section{MATERIAIS E MÉTODOS}

\subsection{Materiais}

A síntese da hidroxiapatita foi realizada pelo processo de reação de estado sólido utilizando como precursores, o hidróxido de cálcio $\left[\mathrm{Ca}(\mathrm{OH})_{2}\right]$ da Fluka/Analytical, com $96 \%$ de pureza e o fosfato monoácido de cálcio $\left(\mathrm{CaHPO}_{4}\right)$ da Sigma/Aldrich com $98 \%$ de pureza, em quantidades estequiométricas obedecendo a reação 1 .

$$
4 \mathrm{Ca}(\mathrm{OH})_{2}+6 \mathrm{CaHPO}_{4} \quad \rightarrow \quad \mathrm{Ca}_{10}\left(\mathrm{PO}_{4}\right)_{2}(\mathrm{OH})_{2}+6 \mathrm{H}_{2} \mathrm{O}
$$

\subsection{Síntese do pó de HAP}

As reações entre os precursores foram realizadas por: mecanoquímico - HAP(A) e calcinação - HAP(B). No processo mecanoquímico descrito na literatura $[25,26]$. Os reagentes utilizados foram homogeneizados e sintetizados em moinho de bolas planetário de alta energia (Fritsch, modelo Pulverisette 5) por 20 horas a $370 \mathrm{rpm}$, com 10 minutos de pausa a cada meia hora de moagem, obedecendo uma razão entre massa dos 
precursores e massa das bolas ( $1 \mathrm{~g}$ de material para cada 4,04 g de bolas), enquanto na calcinação os precursores foram homogeneizados em almofariz de ágata e levado a uma temperatura de calcinação de $1150{ }^{\circ} \mathrm{C}$ por 24 horas, com tratamento térmico a $600^{\circ} \mathrm{C}$ por uma hora em forno resistivo, com taxa de aquecimento e resfriamento de $4^{\circ} \mathrm{C} / \mathrm{min}$ (EDG, modelo F 1800), sendo investigado somente o produto final.

\subsection{Síntese da cerâmica de HAP}

As amostras em pó da hidroxiapatita foram compactados na forma de pastilhas, em moldes de aço inoxidável em formato circular empregando a técnica de prensagem uniaxial em prensa hidráulica, em que a compressão aplicada foi de aproximadamente $210 \mathrm{MPa}$ durante 120 segundos, logo após foram sinterizadas a $900{ }^{\circ} \mathrm{C}$ por 4 horas.

\subsection{Caracterização}

Após obtenção das HAP (A) e (B), as amostras foram caracterizadas por difração raios - X (DRX) em um difratômetro Rigaku, Modelo: Miniflex II, empregando radiação $\mathrm{Cu} \mathrm{K \alpha}(\lambda=1,5418$ Ångstrom) operando em $30 \mathrm{kV} / 15 \mathrm{~mA}$. A intensidade dos picos de difração foi registrada em intervalo de 10 a $80^{\circ} \mathrm{em} 2 \theta$ com passos de $0,02^{\circ}$ a cada 2 segundos. Utilizou-se o banco de dados JCPDS (Joint Committee on Powder Diffraction Standards, Swarthmore, USA) para identificar as fases cristalinas das amostras. Foram utilizados os difratogramas de raios $\mathrm{X}$ para calcular o tamanho médio do cristalito das amostras, por meio da largura a meia altura dos picos de difração e utilizado a equação de Scherrer, apresentada na equação (2):

$$
\mathrm{L}_{\mathrm{c}}=\frac{\mathrm{k} \cdot \lambda}{\beta \cdot \cos (\theta)}
$$

em que, $\mathrm{L}_{\mathrm{c}}$ é o tamanho do cristalito; $k$ é uma constante que depende da forma do cristal, podendo variar entre 0,9 e 1,$0 ; \lambda$ é o comprimento de onda dos raios X; $\theta$ é o ângulo de Bragg do pico difratado e $\beta$ é valor da largura a meia altura do pico de difração correspondente [27,28]. Utilizou-se o software DBWS Tools 2.3 para realizar o refinamento de Rietveld.

As medidas de espectroscopia Raman foram realizadas em espectrômetro triplo da Princeton Trivista 557 operando na configuração subtrativa e equipado com detector CCD (Charge-Coupled Device) resfriado por termoeletricidade. Um laser de íons hélio-neônio operando em $632,8 \mathrm{~nm}$ e potência de $70 \mathrm{~mW}$ foi utilizado como fonte de excitação. As fendas foram ajustadas a fim de se obter uma resolução espectral de $2 \mathrm{~cm}^{-1}$.

As análises morfológicas e as razões de cálcio/fósforo $(\mathrm{Ca} / \mathrm{P})$ foram verificadas por meio da microscopia eletrônica de varredura (MEV) e da espectroscopia de energia dispersiva de raios X (EDX) com o equipamento TESCAN, modelo VEGA3 com um EDX acoplado. Para a quantificação do tamanho médio dos grãos utilizou-se o programa computacional ImageJ, software de domínio público com código aberto, desenvolvido pelo National Institute of Health (NIH), Estados Unidos [29].

As medidas de densidade foram realizadas nas cerâmicas das $\operatorname{HAP}(\mathrm{A})$ e (B) a temperatura ambiente $\left(25^{\circ} \mathrm{C}\right.$ ). Utilizou-se picnômetro com $25 \mathrm{~mL}$ de água destilada ( $\rho$ água $25^{\circ} \mathrm{C}=0,99704 \mathrm{~g} / \mathrm{cm}^{3}$ ), micrômetro da Pan e balança analítica digital Shimadzu modelo AUY 220. As amostras foram imersas em água destilada por 10 minutos para saturação dos poros. Nas medidas de densidade, obedeceu a equação [30]:

$$
\rho_{A}=\frac{m_{A}}{m_{L}-m_{L A}+m_{A}} \times \rho_{L}
$$

em que $\rho_{\mathrm{A}}$ é a densidade da amostra, $\rho_{\mathrm{L}}$ densidade do líquido, $\mathrm{m}_{\mathrm{A}}$ massa da amostra, $\mathrm{m}_{\mathrm{L}}$ massa do picnômetro com o líquido, $\mathrm{m}_{\mathrm{LA}}$ massa do conjunto líquido-amostra.

A densidade relativa entre o valor experimental e o valor teórico pode ser medida pela equação:

$$
(\%) \rho_{\mathrm{AT}}=\frac{\rho_{\mathrm{A}}}{\rho_{\mathrm{T}}} \times 100
$$

na qual $\rho_{\mathrm{T}}$ é a densidade teoria do material.

Para cálculo da porosidade das amostras desta pesquisa, utilizou-se a equação [31]:

$$
(\%) \mathrm{P}=\left[1-\left(\frac{\mathrm{D}}{3,156}\right)\right] \times 100
$$


em que $P$ é a porosidade e $\mathrm{D}$ está relacionado as dimensões das amostras, sendo dado por:

$$
D=\frac{4 \cdot m_{A}}{d^{2} \cdot \pi \cdot h}
$$

onde dé o diâmetro da amostra e h é sua espessura.

As medidas de microdureza foram realizadas por indentações Vickers nas faces das cerâmicas da HAP, usando um padrão testador de microdureza Vickers (SHIMADZU HMV2). As amostras de HAP(A) e HAP(B) foram submetidas a uma carga de 50 gf por $30 \mathrm{~s}$, em cada amostra foram realizadas 3 indentações. A microdureza Vickers (HV) das amostras foi calculada usando o comprimento médio das diagonais, de acordo com a expressão [32]:

$$
\mathrm{Hv}=\frac{\mathrm{L}}{2 \cdot \mathrm{d}^{2}}
$$

onde L é a carga de indentação e d o comprimento médio da diagonal.

\section{RESULTADOS E DISCUSSÃO}

\subsection{Difração de raios X (DRX)}

Os difratogramas (Figura 2) das amostras em pó da hidroxiapatita (HAP) sintetizada pelo método mecanoquímico (A) e por calcinação (B) mostram os picos das fases da HAP $\left[\mathrm{Ca}_{10}\left(\mathrm{PO}_{4}\right)_{6}(\mathrm{OH})_{2}\right]$ bem definidas, comparadas com o padrão JCPDS (74-0565), tanto para a HAP (A) quanto para (B). Na HAP(A) identificamse a formação de única fase de hidroxiapatita, que condiz com difratogramas encontrados por SILVA et al. [25, 26]. Já na $\mathrm{HAP}(\mathrm{B})$, há existência de uma segunda fase identificada, o fosfato tricálcio $\left[\mathrm{Ca}_{3}\left(\mathrm{PO}_{4}\right)_{2}\right]$ (JCPDS 09-0169), sendo um fosfato de cálcio decorrente da alta energia fornecida pelo calor durante a calcinação. É muito comum ocorrer à formação de mais de um fosfato de cálcio na produção de hidroxiapatita, quando seu processo de síntese, for submetido ao tratamento térmico com temperaturas superiores a $700{ }^{\circ} \mathrm{C}$ [33]. Há relatos na literatura da formação de mais de uma fase de fosfato de cálcio, quando ao produzirem a hidroxiapatita, submeteram-na a um tratamento térmico de $1000{ }^{\circ} \mathrm{C}$ e de $950{ }^{\circ} \mathrm{C}[34,35]$. Nota-se que os picos de difração da HAP (B) apresentam um estreitamento de picos, quando comparados ao da HAP (A). Sugerese ser devido ao fato da HAP (B) ter sido sintetizada em altas temperaturas, o que proporcionou um aumento de cristalinidade [16]. Trabalhos como o de GOTO et al. [36] e SCALERA et al. [37] apresentam picos de difratogramas de raios $\mathrm{X}$ estreitos da hidroxiapatita, quando submetidas à influência de calor em suas sínteses, no que corrobora com os difratogramas encontrados neste trabalho. A calcinação dos pós precursores da HAP pode alterar fases do pó obtido, uma vez que as fases de fosfato de cálcio possuem diferentes estabilidades térmicas.

O tamanho médio do cristalito das amostras foi calculado, obtendo valores de $23,35 \pm 0,70 \mathrm{~nm}$ e $47,68 \pm 2,98 \mathrm{~nm}$ para as amostras HAP (A) e (B), respectivamente. Percebe-se que a amostra HAP (B) tem o dobro do tamanho médio do cristalito em relação ao da HAP (A), comprovando o estreitamente da base dos picos de difração devido ao processo térmico ao qual foi submetido. Isso foi observado no trabalho de AZEVEDO et al. [28] ao estudarem o comportamento do tamanho médio do cristalito da hidroxiapatita quando foi submetida a temperaturas de calcinação mais elevadas, encontrando valores variando entre 15 a $55 \mathrm{~nm}$. Já SILVA et al. [26] obtiveram valores do tamanho médio de cristalito variando entre 22 a $39 \mathrm{~nm}$, ao produzirem hidroxiapatita por moagem mecânica reativa de alta energia com diferentes tempos de moagem e reagentes. Os cristalitos determinam orientações na formação dos prismas do esmalte da dentina, uma vez que, o esmalte do dente é formado basicamente por hidroxiapatita e para uma melhor interação com o organismo, tamanhos de cristalitos menores que $100 \mathrm{~nm}$ são os mais desejáveis [38]. MANDAL et al. [39] relatam que o pó de HAP nanocristalino exibe maior área superficial e pode fornecer densificação aprimorada para reduzir a temperatura de sinterização.

O refinamento Rietveld das amostras HAP (A) e (B) apresentado na Figura 3, observam-se as diferenças residuais entre os valores observados e calculados dos padrões de difração. Nota-se que em todos os picos, as diferenças residuais foram pequenas, obtendo resultados satisfatórios. O refinamento também indicou que, para a $\mathrm{HAP}(\mathrm{B})$, a segunda fase de fosfato tricálcio $\left[\mathrm{Ca}_{3}\left(\mathrm{PO}_{4}\right)_{2}\right]$ está presente na amostra em torno de $6,36 \%$, o restante, $93,64 \%$, é referente a hidroxiapatita $\left[\mathrm{Ca}_{10}\left(\mathrm{PO}_{4}\right)_{6}(\mathrm{OH})_{2}\right]$. 
A Tabela 1 apresenta os valores dos parâmetros de redes, o volume da célula unitária e a densidade téorica fornecidos pelo refinamento de Rietveld que estão dentro da faixa considerada aceitável, uma vez que, o fator de perfil ponderado $\left(\mathrm{R}_{\mathrm{wp}}\right)$ e fator de perfil $\left(\mathrm{R}_{\mathrm{P}}\right)$ são índices que melhor refletem a qualidade do refinamento, em termos matemáticos e habitualmente são encontrados na literatura entre 10 a $20 \%$ [40, 41]. Os índices residuais (S) são perfeitos quando mais próximo a 1, todavia na prática os valores inferiores a 5 caracterizam um refinamento otimizado [42]. Os parâmetros de rede a, b e c das $\operatorname{HAP}(\mathrm{A})$ e (B) possuem valores semelhantes, assim como os volumes das células unitárias e as densidades teóricas. Os resultados encontrados corroboram com os valores determinados por SARKAR e KANAM [40]. Por sua vez, os valores de $\mathrm{R}_{\mathrm{wp}}$ estão abaixo dos encontrados por ARAÚJO et al. [42], na qual determinaram valores de 15,8 e $16,3 \%$ ao realizar o refinamento da hidroxiapatita utilizando programas de softwares diferentes.

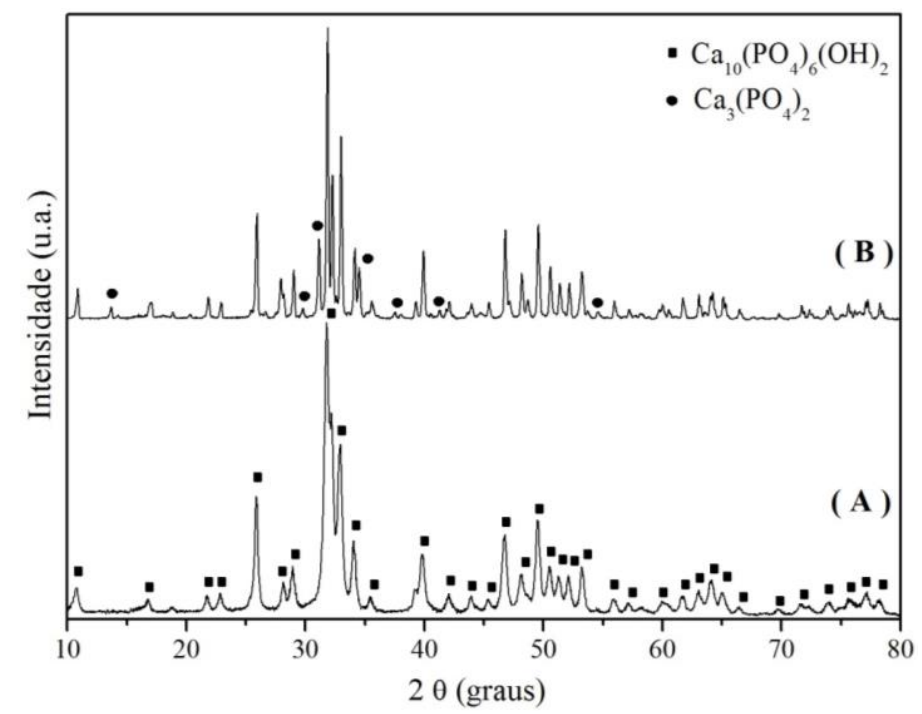

Figura 2: Difratogramas das amostras de hidroxiapatita (HAP) sintetizadas por mecanoquímica (A) e por calcinação (B).

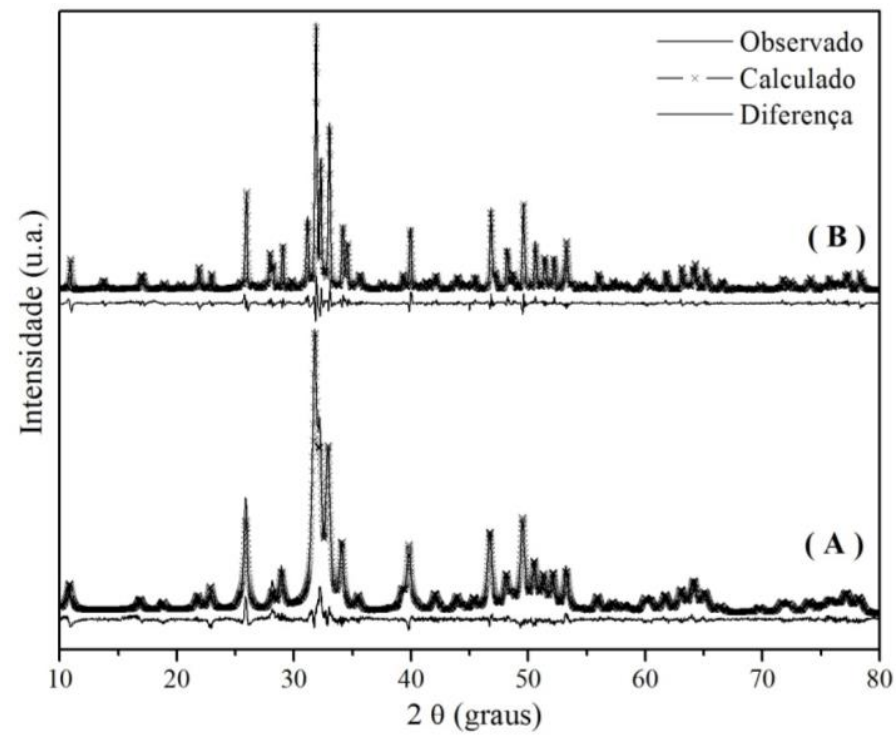

Figura 3: Diferenças residuais das amostras de hidroxiapatita (HAP) sintetizadas por mecanoquímica (A) e por calcinação (B), obtidas pelo refinamento Rietveld. 
Tabela 1: Parâmetros de refinamento das amostras de hidroxiapatita (HAP) sintetizadas por mecanoquímica (A) e por calcinação (B) obtidos pelo método Rietveld.

\begin{tabular}{|c|c|c|c|c|c|c|c|c|}
\hline HAP & $\begin{array}{c}\text { Resíduo } \\
\text { (S) }\end{array}$ & $\begin{array}{l}\mathbf{R}_{\mathrm{wp}} \\
(\%)\end{array}$ & $\begin{array}{l}\mathbf{R}_{\mathrm{p}} \\
(\%)\end{array}$ & $\begin{array}{c}\mathbf{a} \\
(\hat{A})\end{array}$ & $\begin{array}{c}\mathbf{b} \\
(\hat{A})\end{array}$ & $\begin{array}{c}\text { c } \\
(\hat{A})\end{array}$ & $\begin{array}{c}\text { Volume } \\
\left(\AA^{3}\right)\end{array}$ & $\begin{array}{c}\text { Densidade } \\
\left(\mathbf{g} / \mathbf{c m}^{3}\right)\end{array}$ \\
\hline A & 1,38 & 10,40 & 7,87 & 9,4202 & 9,4202 & 6,8769 & 528,4984 & 3,158 \\
\hline $\mathrm{B}$ & 1,64 & 12,78 & 9,55 & 9,4125 & 9,4125 & 6,8807 & 527,9263 & 3,161 \\
\hline
\end{tabular}

\subsection{Espectro Raman}

A Figura 4 apresenta os espectros Raman da HAP (A) e (B) com as bandas de identificações das vibrações das moléculas. A identificação dos modos vibracionais foram realizadas mediante os relatos de trabalhos da literatura [26, 43-47] e estão apresentados na Tabela 2. Nesses espectros foram possíveis perceber e identificar as bandas características da hidroxiapatita em $969 \mathrm{~cm}^{-1}$, sendo que na HAP (B) há o surgimento de novas bandas vibracionais em 1375 e $1405 \mathrm{~cm}^{-1}$.

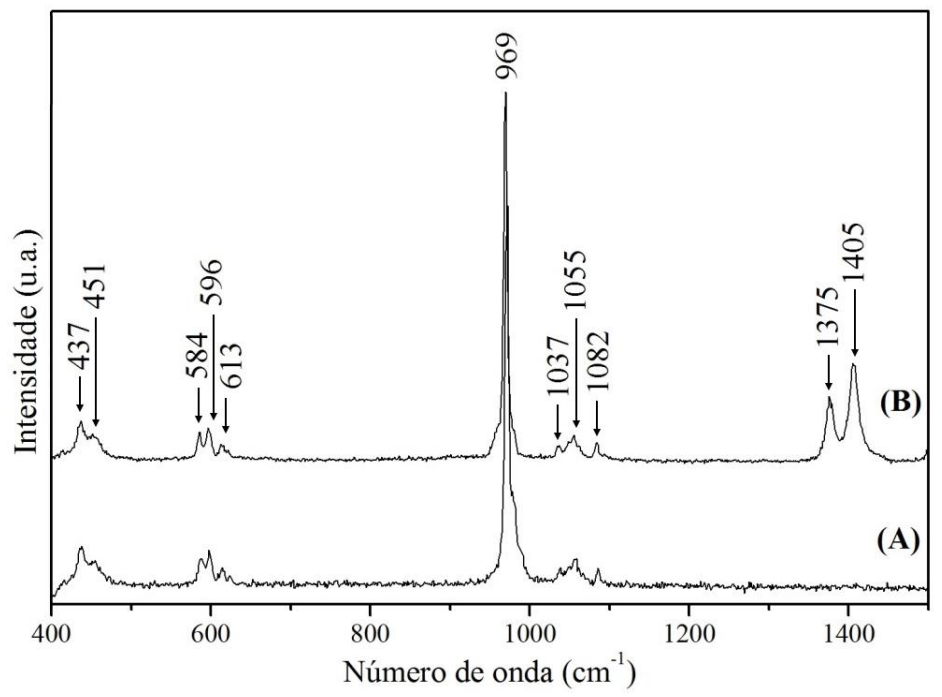

Figura 4: Espectro Raman da HAP obtido pelos métodos mecanoquímico (A) e calcinação (B).

Tabela 2: Modos vibracionais dos espectros Raman das HAP (A) e (B).

\begin{tabular}{c|c|c|c}
\hline Número de onda $\left(\mathbf{c m}^{-\mathbf{1}}\right)$ & Grupo Característico & Modo vibracional & Intensidade \\
\hline 437 & $\mathrm{O}-\mathrm{P}-\mathrm{O}$ & Torção & Forte \\
\hline 451 & $\mathrm{O}-\mathrm{P}-\mathrm{O}$ & Torção & Forte \\
\hline 584 & $\mathrm{O}-\mathrm{P}-\mathrm{O}$ & Estiramento simétrico & Forte \\
\hline 596 & $\mathrm{O}-\mathrm{P}-\mathrm{O}$ & Estiramento simétrico & Forte \\
\hline 613 & $\mathrm{O}-\mathrm{P}-\mathrm{O}$ & Estiramento simétrico & Forte \\
\hline 969 & $\mathrm{P}-\mathrm{O}-\mathrm{P}$ & Estiramento simétrico & Muito forte \\
\hline 1037 & $\mathrm{P}-\mathrm{O}$ & Estiramento antissimétrico & Forte \\
\hline 1055 & $\mathrm{P}-\mathrm{O}$ & Estiramento antissimétrico & Ombro \\
\hline 1082 & $\mathrm{P}-\mathrm{O}$ & Estiramento antissimétrico & Ombro \\
\hline 1375 & $\mathrm{CO}_{3}^{2-}$ & Estiramento simétrico fora do plano & Forte \\
\hline 1405 & $\mathrm{CO}_{3}^{2-}$ & Estiramento simétrico fora do plano & Forte \\
\hline & \multicolumn{2}{|}{}
\end{tabular}

As bandas nas regiões de 437 e $451 \mathrm{~cm}^{-1}$ podem ser atribuídas a vibração $\mathrm{O}-\mathrm{P}-\mathrm{O}$, sendo estes associados a uma torção de intensidade forte. Os modos vibracionais obtidos na região entre 584, 596 e 613 $\mathrm{cm}^{-1}$ representam um estiramento antissimétrico de intensidade forte para as ligações $\mathrm{O}-\mathrm{P}-\mathrm{O}$. A banda em torno de $969 \mathrm{~cm}^{-1}$ representa um estiramento simétrico de intensidade muito forte, este pico é o mais 
característico da hidroxiapatita. Modos de vibrações associados à ligação $\mathrm{P}-\mathrm{O}$ são observadas na região de 1037, 1055 e $1082 \mathrm{~cm}^{-1}$, sendo que $1037 \mathrm{~cm}^{-1}$ tem intensidade forte e os demais representam um ombro.

O ponto interessante para a amostra HAP (B) é o surgimento das bandas em torno de $1375 \mathrm{e} 1405 \mathrm{~cm}^{-}$ ${ }^{1}$ que podem ser característicos de absorção de $\mathrm{CO}_{3}{ }^{2-}$, uma vez que nos precursores pode haver presença de carbono, devido ao fato de o hidróxido de cálcio $\left[\mathrm{Ca}(\mathrm{OH})_{2}\right]$ utilizado ter $96 \%$ de pureza, possibilitando $4 \%$ de impureza de outros elementos. Outra possibilidade é o fator de que no processo de síntese da HAP (B) a calcinação a altas temperaturas por muito tempo em ambiente de gás não inerte possibilita a interação do gás com os reagentes, ocorrendo substituições de íons de carbonatos $\left(\mathrm{CO}_{3}{ }^{2-}\right)$ no tetraédrico do fosfato $\left(\mathrm{PO}_{4}{ }^{3-}\right)$, dando chances de formar a hidroxiapatita carbonatada do Tipo A [44,45]. Já GOUVEIA [48], relata que pode ocorrer substituição de sítios de hidroxila $\left(\mathrm{OH}^{-}\right)$por carbonato $\left(\mathrm{CO}_{3}{ }^{2-}\right)$, dando origem a hidroxiapatita carbonatada do Tipo B. É comum encontrar carbonatos em ossos de indivíduos, sendo que sua quantidade varia entre 4 a $8 \%$ em massa. O carbonato tem um papel importante em apatitas, pois aumenta sua reatividade, aumentando a taxa de dissolução em ácidos e no produto de solubilidade [48].

ANTONAKOS et al. [45] relatam a presença de bandas de $\mathrm{CO}_{3}{ }^{2-}$ na estrutura da hidroxiapatita na região próxima a 1396 e $1439 \mathrm{~cm}^{-1}$, porém com intensidade baixa. SOFRONIA et al. [46] trabalharam com hidroxiapatita de osso bovino, calcinada a $900{ }^{\circ} \mathrm{C}$ por uma hora e o espectro Raman mostrou a presença de bandas com baixa intensidade na região próxima a $1400 \mathrm{~cm}^{-1}$. Nesta pesquisa, observa-se que na região próxima a $1400 \mathrm{~cm}^{-1}$, a intensidade das bandas são fortes, tendo como possível causa, a alta temperatura e o tempo longo de calcinação. A espectroscopia Raman evidenciou a presença de carbonato na estrutura de hidroxiapatita, quando sintetizada por calcinação.

\subsection{Microscopia Eletrônica de Varredura (MEV)}

As micrografias das amostras cerâmicas sinterizadas das HAP (A) e (B) são apresentadas nas Figuras 5 (a) e (b) detalhando a morfologia dos pós compactados uniaxialmente na forma de disco. Na Figura 5 (a), observase que a $\mathrm{HAP}(\mathrm{A})$ produzida por mecanoquímica apresenta os grãos mais uniformes e tamanhos nanométricos, variando entre $285 \mathrm{~nm}$ a $2,73 \mu \mathrm{m}$, tendo uma média de $619 \mathrm{~nm}$. YEONG et al. [22] foram pioneiros na síntese de hidroxiapatita via mecanoquímica, na qual relatam que a compressão de alto impacto e o aumento instantâneo da temperatura local nos pontos de colisão são fatores principais que contribuem para o desencadeamento da reação de estado sólido em escala nanométrica. Imagens de microscopia da hidroxiapatita comercial são mostradas no trabalho de CAPANEMA [49] e são semelhantes ao da Figura 5 (a). Sugere-se que a síntese em moinho de alta energia favorece a uniformidade e diminuição dos tamanhos de grãos. Isso é relatado por MANDAL et al. [39] que menciona a diminuição do tamanho de grão e do tamanho do cristalito à medida que se aumenta o tempo de moagem, quando analisou a otimização de parâmetros da hidroxiapatita sintetizada em moinho planetário de acionamento duplo, por tempo de moagem mecânica e razão dos diâmetros das bolas. LALA et al. [19] cita que a HAP em escala nanométrica melhora caraterísticas como: bioatividade, osteointegratividade, atividade catalítica e capacidade de adsorção. Já na HAP (B) (Figura 5b), observam-se grãos com tamanhos diversificados e formas irregulares quando comparados com a HAP (A), (Figura 5a). Em algumas regiões, há existência de aglomerados grãos, devido à possibilidade de ter ocorrido soldagem de fusão do material durante a calcinação. Os grãos da HAP (B) são maiores, variando entre 0,38 a 5,86 $\mu \mathrm{m}$, tendo em média tamanho de $908 \mathrm{~nm}$. Este aumento do tamanho de grãos pode estar associado à calcinação em $1150{ }^{\circ} \mathrm{C}$, uma vez que o tamanho dos grãos tende a crescer quando aquecidos a altas temperaturas. Trabalhos como de GOTO et al, e SCALERA et al., [36, 37] relatam crescimento de tamanho de grãos à medida que aumentou a temperatura no processo de calcinação de síntese da hidroxiapatita. As formas irregulares podem ter ocorrido devido à homogeneização dos reagentes ter sido realizada em almofariz de ágata, favorecendo uma distribuição irregular de grãos. 

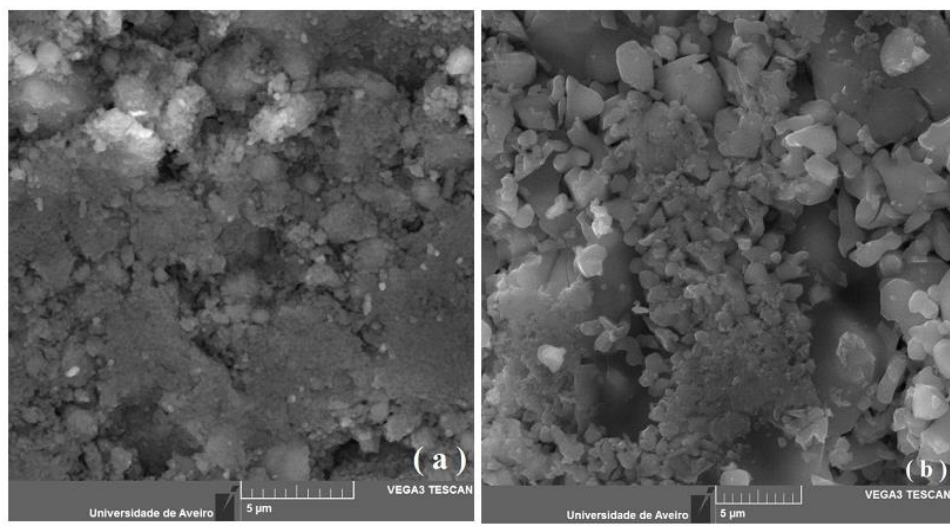

Figura 5: Micrografia eletrônica de varredura da (a) HAP (A) e (b) da HAP (B)

SILVA et al. [25] determinou o tamanho médio dos grãos da hidroxiapatita sintetizada pelo método mecanossíntese, em diferentes tempos de moagem, conseguindo valores em escala nanométrica, semelhante à escala encontrada na Figura 5 (a). Micrografias análogas a da Figura 5 (b) foram observadas por SILVA et al. [50], ao produzirem hidroxiapatita por outro método de reação de estado sólido através de um aparelho de micro-ondas adaptado com uma célula susceptora. Os autores apresentaram grãos com tamanhos micrométricos e formas diversificadas. Em consonância, HUI et al. [35] encontraram valores de tamanhos de grãos também em escala micrométricas, variando entre 3 a $5 \mu \mathrm{m}$, ao utilizarem o método hidrotermal na síntese da hidroxiapatita. A comparação desses resultados sugere que os métodos aplicados neste trabalho, nas sínteses das HAP(A) e (B), apresentam equivalência na morfologia da hidroxiapatita sintetizadas por outros trabalhos encontrados na literatura.

A espectroscopia de energia dispersiva de raios $\mathrm{X}$ (EDX) foi utilizada para análises quantitativas composicional das amostras HAP (A) e (B), com o intuito de verificar se os valores experimentais das razões molares $\mathrm{Ca} / \mathrm{P}$ condizem com os valores teóricos da hidroxiapatita. As razões $\mathrm{Ca} / \mathrm{P}$ obtidas pelo EDX foram de 1,71 e 1,87 para a HAP $(\mathrm{A})$ e $(\mathrm{B})$, respectivamente. Essas diferenças de valores podem ser relacionadas à área selecionada aleatoriamente durante as medidas do EDX, sendo que na área selecionada poderia conter mais cálcio $\left(\mathrm{Ca}^{2+}\right)$ do que fósforo $\left(\mathrm{P}^{5+}\right)$, dando a diferença de valores entre as razões molares $\mathrm{Ca} / \mathrm{P}$. Outra possibilidade está associada ao fato da HAP (B) apresentar mais uma fase de fosfato de cálcio, podendo favorecer quantidade relativamente maior de cálcio $\left(\mathrm{Ca}^{2+}\right)$ do que de fósforo $\left(\mathrm{P}^{5+}\right)$. Como o espectro Raman apresentou bandas relacionadas a carbonatos $\left(\mathrm{CO}_{3}{ }^{2-}\right)$ na $\mathrm{HAP}(\mathrm{B})$, também pode-se relacionar a diferença de razão molar $\mathrm{Ca} / \mathrm{P}$ ao carbonato, uma vez que, substituições de carbonatos $\left(\mathrm{CO}_{3}{ }^{2-}\right)$ em sítios de tetraédrico do fosfato $\left(\mathrm{PO}_{4}{ }^{3-}\right)$ ou de hidroxila $\left(\mathrm{OH}^{-}\right)$alteram a razão $\mathrm{Ca} / \mathrm{P}$ em apatitas ou mudança em suas fórmulas química [48]. Esses valores são próximos ao valor teórico da hidroxiapatita $(\mathrm{Ca} / \mathrm{P}=1,67)$ e compatíveis aos valores já encontrados na literatura. Silva et al. [50] que obteve valores de razões $\mathrm{Ca} / \mathrm{P}$ iguais a 1,72; 1,73; 1,74 e 1,79 para diferentes procedimentos de tratamento térmico e tempo de exposição à radiação de microondas. LIU et al. [16], AZEVEDO et al. [28] e RAYNAUD et al. [51], apresentaram razões de Ca/P com valores próximos aos valores encontrados neste trabalho, variando entre 1,5 a 1,73, estando dentro da faixa $(1,5$ a 2,0) considerada como hidroxiapatita, conforme a literatura $[3,10]$.

\subsection{Densidade e porosidade}

Na tabela 3, têm-se os valores das densidades e porosidades das cerâmicas sinterizadas das hidroxiapatitas HAP(A) e (B). Nota-se que os valores das densidades experimentais ficaram próximos ao valor teórico, obtendo uma relação de mais de $82 \%$, para HAP(A) e mais de $83 \%$ para HAP(B). Os resultados das densidades experimentais corroboram com valor encontrado por MONTEIRO [52], em que o mesmo determinou em $2,76 \mathrm{~g} / \mathrm{cm}^{3}$ a densidade da hidroxiapatita com $99 \%$ de pureza.

Tabela 3: Densidades e porosidades da hidroxiapatita HAP(A) e (B).

\begin{tabular}{c|c|c|c|c}
\hline \multirow{2}{*}{ HAP } & \multicolumn{3}{|c|}{ DENSIDADE } & \multirow{2}{*}{ POROSIDADE } \\
\cline { 2 - 4 } & Experimental $\mathbf{( g / \mathbf { c m } ^ { 2 } )}$ & Teórica $\left(\mathbf{g} / \mathbf{c m}^{\mathbf{2}}\right)$ & Relativa (\%) & 32,82 \\
\hline A & 2,614 & 3,16 & 82,72 & 25,44 \\
\hline B & 2,652 & 3,16 & 83,92 & \\
\hline
\end{tabular}


É possível aproximar o valor da densidade experimental da hidroxiapatita com o valor teórico, para tanto, devemos modificar métodos experimentais em sua produção, tais como; aumentar o tempo de moagem, para diminuir o tamanho do grão, o que facilitaria a compactação da amostra; aumentar a pressão aplicada na formação das pastilhas (corpo de prova), para se obter maior compactação do pó; aumentar o tempo de sinterização do material, para que aumente o tamanho do grão e diminua os espaços vazios entre eles e obter um material mais denso.

O percentual da porosidade das $\operatorname{HAP}(\mathrm{A})$ e (B) apresentaram valores considerados satisfatórios, pois estão próximos aos encontrados na literatura [31,53]. ROSA et al. [31] avaliaram a porosidade da hidroxiapatita comercial prensada uniaxialmente, na qual variaram pressão, temperatura e tempo de sinterização, encontrando valores que variaram entre 10 e $35 \%$ de porosidade. A matriz porosa de biocerâmicas tem aplicações interessantes na área biomédica, tais como: incorporação de radionuclídeos para tratamentos terapêuticos de câncer; facilitação na penetração de fluidos corporais, essenciais para o crescimento de tecido ósseo e maior degradação em meio fisiológico [19,54]. Porém a alta porosidade pode promover características indesejáveis, tais como a osteogênese, conhecida como "ossos de vidro", ou seja, fragilidade óssea. A densidade e a porosidade são características intrínsecas de materiais cerâmicos e viabilizam propriedades de adesão do material ao tecido imunológico.

\subsection{Microdureza Vickers}

As medidas de microdureza Vickers determinadas nas cerâmicas sinterizadas da hidroxiapatita $\mathrm{HAP}(\mathrm{A})$ e (B) foram 1290,4 $\pm 0,54 \mathrm{MPa}$ e $461,78 \pm 0,36 \mathrm{MPa}$, respectivamente. Nota-se que os valores obtidos mostram uma grande diferença, sendo a HAP(A) quase 3 vezes maior que a HAP(B). O que pode estar associado à mudança de morfologia superficial apresentada pelas amostras sintetizadas, que é comentada por Filho e colaboradores [32] encontraram valor superior a $1300 \mathrm{MPa}$ na sínteses da hidroxiapatita realizada pelo método de moagem mecânica. Outro fator que pode estar pertinente a essa diferença de microdureza é uma possível maior compactação dos grãos da $\mathrm{HAP}(\mathrm{A})$ em relação a $\mathrm{HAP}(\mathrm{B})$, uma vez que apresenta grãos de tamanho médio menores que a HAP(B). Mandal et al. [39] alude que a dureza e tenacidade à fratura podem ser melhoradas na cerâmica de HAP, quando a mesma apresenta escala nanométrica. Machado e colaboradores [55] encontraram resultados de microdureza Vickes correspondentes a 35,84 HV (351,54 MPa) para hidroxiapatita sintetizada a partir da casca de ovo e 40,86 HV (400,71 MPa) para uma hidroxiapatita comercial, ambas com aplicação de tensão média de $216,25 \mathrm{Mpa}$ por 20 min e sinterizadas a $1000{ }^{\circ} \mathrm{C}$. Esses valores, apesar de mostrar superioridade no tempo de conformação e na temperatura de sinterização, são próximos ao valor da HAP(B) sintetizada neste trabalho. Medidas de microdureza são informações necessárias para resistência à deformação plástica, desgaste, corte e risco ou penetração de um material em outro [56].

\section{CONCLUSÕES}

Os diferentes métodos empregados para a síntese do pó de hidroxiapatita indicaram ser eficazes e confiáveis. A moagem mecânica de alta energia mostrou ser mais eficiente pelo fato de formar apenas uma fase cristalográfica da hidroxiapatita e apresenta grãos mais uniformes e menores. A calcinação pode ser considerada um método mais simples para síntese da hidroxiapatita, todavia forma mais de uma fase cristalográfica de fosfato de cálcio e apresenta grãos maiores com tamanhos irregulares, diferindo da síntese por moagem. A difração de raios X da HAP (A) e (B) verificou a fase cristalográfica da hidroxiapatita, mostrando uma boa cristalinidade, com cristalitos em escalas nanométricas confirmados no refinamento Rietveld. Os espectros Raman e análises comparativas com a literatura reforçam os resultados das sínteses das HAP (A) e (B) obtidas nas difrações de raios X. A microscopia eletrônica de varredura evidenciou que há diferenças de tamanhos de grãos entre as HAP (A) e (B) em métodos diferentes de síntese. Na espectroscopia de energia dispersiva de raios $\mathrm{X}$, a razão $\mathrm{Ca} / \mathrm{P}$ confirmou ser hidroxiapatita para as amostras $\mathrm{HAP}(\mathrm{A})$ e HAP(B), pois estão dentro da faixa etária do valor teórico. As medidas de densidades foram satisfatórias, uma vez que, apresentou valores próximos ao valor teório e densidades relativas acima de $80 \%$. Os valores para a porosidade foram aceitáveis, pois baixa porosidade, pode ocasionar baixa biodegradação da amostra em meio fisiológico e diminui a possibilidade de promover osteogênese (fragilidade óssea). As medidas de microdureza se mostraram muito dinâmicas, em relação às propriedades mecânicas, na qual apresentou boa resistência à deformação plástica.

\section{AGRADECIMENTOS}

Os autores agradecem à Fundação de Amparo à Pesquisa e ao Desenvolvimento Científico e Tecnológico do 
Maranhão (FAPEMA) que financiou parte desta pesquisa. Ao Programa de Pós-graduação em Ciência dos Materiais da Universidade Federal do Maranhão. Ao Departamento de Física (I3N) da Universidade de Aveiro, Portugal, pela utilização de seus laboratórios.

\section{BIBLIOGRAFIA}

[1] CAO, W.; HENCH, L. L., "Bioactive materials", Ceramics International, v. 22, pp. 493-507, 1996.

[2] LEMAITRE, J.; MIRTCHI, A.; MORTIER, A., "Calcium phosphate cement for medical use: state of the art and perspectives of development", Ceramics Science and Tchnological, v. 52, pp. 141-146, 1987.

[3] RIGO, E. C. da S., GEHRKE, S. A., CARBONARI, M., "Síntese e caracterização de hidroxiapatita obtida pelo método de precipitação", Revista Dental Press de Periodontia Implantol, Maringá, vol. 1, n. 3, pp. 39-50, jul/ago/set. 2007.

[4] WANG, M. "Developing bioactive composite materials for tissue replacement", Biomaterials, v. 24 (13) pp. 2133-2151, 2003.

[5] BONAN, R. F., BONAN, P. R. F., BATISTA, A. U. D., et al., "Métodos de reforço microestrutural da hidroxiapatita", Cerâmica, v. 60, pp. 402-410, 2014.

[6] SYGNATOWICZ, M., KEYSHAR, K., TIWARI, A., "Antimicrobial Properties of Silver-doped Hydroxyapatite nano-powders and Thin Films", Biological Biomedical Materials, v. 62, pp. 65-70, 2010.

[7] SANTOS, M. V. B., OSAJIMA, J. A., SILVA-FILHO, E. C., "Hidroxiapatita: suporte para liberação de fármacos e propriedades antimicrobianas", Cerâmica, v. 62, pp. 256-265, 2016.

[8] PRAMANIK, S., PRAMANIK, A. K., RAI, X K.N., GARG, A., "Development of high strength hydroxyapatite by solid-state-sintering process", Ceramics International, v. 33, pp. 419-426, 2007.

[9] PARRIS, G. E., ARMOR, J. N., "Catalytic Cracking of organic amides. I Production of Nvinylformamide", Applied Catalysis, v. 78, n. 1, pp. 45-64, 1991.

[10] GUASTALDI, A. C.; APARECIDA, A. H., "Fosfatos de cálcio de interesse biológico: importância como biomateriais, propriedade e métodos de obtenção de recobrimentos", Química Nova, v. 33, n. 6, pp. 1352$1358,2010$.

[11] FIHRI, A., LEN, C., VARMA, R. S., SOLHY,A., "Hydroxyapatite: A review of sytheses, structure and applications in heterogeneous catalysis", Coordination Chemistry Reviews, v. 347, pp.48-76, 2017.

[12] LAURENCIN, D., ALMORA-BARRIOS, N., LEEUW, N. H., et al., "Magnesium incorporation into hydroxyapatite", Biomaterials, v.32, pp.1826-1837, 2011.

[13] VIANA, J. R., "Síntese e caracterização de hidroxiapatita dopada com terras raras (Eu3+ e Yb3+) com propriedade luminescentes”, Dissertação de M., UFMA, Imperatriz - MA, 2015.

[14] SANTOS, L. A.., Cimento de fosfato de cálcio reforçado por fibras, São Paulo, Blucher Acadêmico, 2008.

[15] RIGO, E.C.S., OLIVEIRA, L.C., SANTOS, L.A., BOSCH, A.O., CARRODEGUAS, R.G., "Implantes metálicos recobertos com hidroxiapatita", Revista Brasileira de Engenharia Biomédica, v. 15, n. 1-2, pp. 2129, 1999.

[16] LIU, H. S., CHIN, T. S., LAI, L. S., et al., "Hydroxyapatite synthesized by a simplified hydrothermal method", Ceramics International, v. 23, pp. 19-25, 1997.

[17] NASIRI-TABRIZI, B., FAHAMI, A., EBRAHIMI-KAHRIZSANGI, R., "Effect of milling parameters on the formation of nanocrystalline hydroxyapatite using different raw materials", Ceramics International, v. 39, pp. 5751-5763, 2013.

[18] RHEE, S. H., "Synthesis of hydroxyapatite via mechanochemical treatment", Biomaterials, v. 23, pp. 1147-1152, 2002.

[19] LALA, S., SATPATI, B., KAR, T., PRADHAN, S.K., "Structural and microstructural characterizations of nanocrystalline hydroxyapatite synthesized by mechanical alloying", Materials Science and Engineering C, v. 33, pp. 2891-2898, 2013.

[20] SURYANARAYANA, C., IVANOV, E., BOLDYREV, V.V., "The science and technology of mechanical alloying", Materials Science and Engineering A, 304-306, pp. 151-158, 2001.

[21] ZADOROZHNYY, V.Y., KAEVITSER, E.V., KOPYLOV, A.N., et al., "Synthesis of the hydroxyapatite coatings on the Ti substrates by mechanical alloying", Surface \& Coatings Technology, v. 281, pp. 157163,2015 . 
[22] YEONG, K. C. B., WANG, J., NG, S.C., "Mechanochemical synthesis of nanocrystalline hydroxyapatite from $\mathrm{CaO}$ and $\mathrm{CaHPO}_{4}$ ", Biomaterials, v. 22, pp. 2705-2712, 2001.

[23] GUO, X., YAN, H., ZHAO, S., et al., "Effect of calcining temperature on particle size of hydroxyapatite synthesized by solid-state reaction al room temperature". Advanced Powder Techology, v. 24, pp. 1034-1038, 2013.

[24] GUIMARAES, J. E. P., A indústria de cal no Brasil. ABPC. São Paulo, 1990.

[25] SILVA, C. C., THOMAZINI, D., PINHEIRO, A. G., et al., "Optical properties of hydroxyapatite obtained by mechanical alloying", Journal of physics and chemistry of solids, v. 63, pp. 1745-1757, 2002.

[26] SILVA, C. C., PINHEIRO, A. G., MIRANDA, M. A. R., et al., "Structural properties of hydroxyapatite obtained by mechanosynthesis", Solid state sciences, v. 5, pp. 553-558, 2003.

[27] SILVA, C. C., GRAÇA, M. P. F., VALENTE, M. A., et al., "Crystallite size study of nanocrystalline hydroxyapatite and ceramic system with titanium oxide obtained by dry Ball milling", Journal. Materils Science, v. 42, pp. 3851-3855, 2007.

[28] AZEVEDO, A. G. S., STRECKER, K., ARAÚJO-JUNIOR, A. G. A., "Avaliação da influência da razão $\mathrm{Ca} / \mathrm{P}$ nos valores de cristalinidade e crescimento de cristais durante a sinterização de pós de hidroxiapatita" Revista Eletrônica de Materiais e Processos, v. 9, n. 3, pp. 125-129, 2014.

[29] MARCOMINI, R. F., SOUZA, D. M. P. F., "Caracterização microestrutural de materiais cerâmicos utilizando o programa de processamento digital de imagens ImageJ”, Cerâmica, v.57, pp.100-105, 2011.

[30] SILVA, A. G. P., Estrutura e propriedades de materiais cerâmicos, Notas de aula, Universidade Estadual do Norte Fluminese, 2008.

[31] ROSA, A. L., SHAREEF, M. Y., NOORT, R. V., "Efeitos das condições de preparação e sinterização sobre porosidade da hidroxiapatita", Pesquisa Odontol Bras, v. 14, n. 3, pp. 273-277, 2000.

[32] FILHO, F. P., NOGUEIRA, R. E. F. Q., GRAÇA, M. P. F., et al., "Structural and mechanical study of the sintering effect in hydroxyapatite doped with iron oxide", Physica B, n. 403, pp. 3826-3829, 2008.

[33] RAYNAUD, S., CHAMPION, E., BERNACHE-ASSOLLAT, D., "Calcium phosphate apatites with variable $\mathrm{Ca} / \mathrm{P}$ atomic ratio II. Calcination an sintering”, Biomaterials, v. 23, pp. 1073-1080, 2002.

[34] GRAEVE, O. A., KANAKALA, R., MADADI, A., et al., "Luminescence variations in hydroxyapatites doped with $\mathrm{Eu}^{2+}$ and $\mathrm{Eu}^{3+}$ ions", Biomaterials, v. 31, pp. 4259-4267, 2010.

[35] HUI, P., MEENA S. L., AGARAWAL, R. D., et al., "Synthesis of hydroxyapatite Bio-ceramic Powder by Hydrothermal Method", Journal of Minerals Characterization \& Engineering, v. 9, n. 8, pp. 683-692, 2010.

[36] SCALERA, F., GERVASO, F., SANOSH, K. P., et al., "Influence of the calcination temperature on morphological and mechanical properties of highly porous hydroxyapatite scaffolds", Ceramics International, v. 39, pp. 4839-4846, 2013.

[37] GOTO, T., SASAKI, K., "Effects of trace elements in fish bonés on Crystal characteristics of hydroxyapatite obtained by calcination”, Ceramics International, v. 40, pp. 10777-10785, 2014.

[38] BARBOSA, M. C., "Síntese de hidroxiapatita por via úmida assistida por métodos físicos", Trabalho de conclusão de curso. Universidade do Vale do Paraíba, 2014.

[39] MANDAL, T., MISHRA, B. K., GARG, A., CHAIRA, D. "Optimization of milling parameters for the mechanosynthesis of nanocrystalline hydroxyapatite", Powder technology, v. 253, pp. 650-656, 2014.

[40] SARKAR, A., KANNAN, S., "In situ synthesis, fabrication and Rietveld refinement of the hydroxyapatite/titania composite coatings on 316 L SS", Ceramics International, v. 40, pp. 6453-6463, 2014.

[41] GOBBO, L. A. "Aplicação da difração de raios - X e método de Rietveld no estudo de cimento Portland”, Tese de D.Sc., Instituto de Geociência-USP, São Paulo, 2009.

[42] ARAÚJO, J. C., SENA, L., BASTOS, I. N., et al., "Síntese da Hidroxiapatita e refinamento estrutural por difração de raios - X", Química Nova, v. 30, n. 8, pp. 1853-1859, 2007.

[43] SINGH, B., DUBEY, A. K., KUMAR, S., et al., "In vitro biocompatibility and antimicrobial activity of wet chemically prepared $\mathrm{Ca}_{10-\mathrm{x}} \mathrm{Ag}_{\mathrm{x}}\left(\mathrm{PO}_{4}\right)_{6}(\mathrm{OH})_{2}(0,0 \leq \mathrm{x} \leq 0,5)$ hydroxyapatites", Materials Science and Engineering C, v. 31, pp. 1320-1329, 2011. 
[44] BERTOLUZZA, A., BOTTURA, G., TADDEI, P., et al., "Vibrational Spectra of Controlled-S tructure Hydroxyapatite Coatings Obtained by the Polymeric Route", Journal of Raman Spectroscopy, v. 21, pp. 759764, 1996

[45] ANTONAKOS, A. LIAROKAPIS, E. LEVENTOURI, T. "Micro-Raman and FTIR studies of synthetic and natural apatites", Biomaterials, v. 28, pp. 3043-3054, 2007.

[46] SOFRONIA, A. M., BAIES, R., ANGHEL, E. M., et al., "Thermal and structural characterization of synthetec and natural nanocrystalline hydroxyapatite", Materials Science and Engineering C, v. 43, pp. 153$163,2014$.

[47] SILVA, C. C., SOMBRA, A. S. B., "Raman spectroscopy measurements of hydroxyapatite obtained by mechanical alloying", Journal of physics and chemistry of solids, v. 65, pp. 1031-1033, 2004.

[48] GOUVEIA, D. S., "Obtenção de pós nanométricos de hidroxiapatita sintetizados com magnésio utilizando ultra-som", Tese de D.Sc., USP, São Paulo - SP, 2008.

[49] CAPANEMA, N. S. V., "Síntese e caracterização de biocerâmicas à base de fosfato de cálcio modificada com nióbio", Dissertação de M. Sc., UFMG. Belo Horizonte, Minas Gerais, Brasil, 2014.

[50] SILVA, C. C., GRAÇA, M. P. F., SOMBRA, A.S.B., et al., "Structural and electrical study of calcium phosphate obtained by a microwave radiation assisted procedure", Physica B, v. 404, pp. 1503-1508, 2009.

[51] RAYNAUD, S., CHAMPION, E, BERNACHE-ASSOLLAT, D., et al., "Calcium phosphate apatites with variable $\mathrm{Ca} / \mathrm{P}$ atomic ratio I Synthesis, characterisation and thermal stability of powders", Biomaterials, v. 23, pp. 1065-1072, 2002.

[52] MONTEIRO, T. F., Caracterização de biocerâmica à base de hidroxiapatite. Dissertação de M. Universidade de Aveiro, Aveiro, Portugal, 2015.

[53] POURDANESH, F., JEBALI, A., HEKMATIMOGHADDAM, S., et al., "In vitro and in vivo evaluation of a new nanocomposite, containing high density polyethylene, tricalcium phosphate, hydroxyapatite, and magnesium oxide nanoparticles", Materials Science an Engineering C, v. 40, pp. 382-388, 2014.

[54] LACERDA, K. A., LAMEIRAS, F. S., SILVA, V. V. "Síntese e caracterização de matrizes porosas de hidroxiapatita para aplicação como fontes radioativas em braquiterapia", Metalurgia \& Materiais, v. 59, pp. 217-223, 2006.

[55] MACHADO, M. V. F., CAMPOS, J. B., AGUILAR, M. S., et al., "Caracterização Mecânica e Microestrutural em Hidroxiapatita Comercial e Sintetizada pelo Método Sol-Gel Utilizando Casca de Ovo de Galinha Como Precursor", In: XXI Encontro Nacional de Modelagem Computacional e IX Encontro de Ciência e Tecnologia de Materiais. Búzios-RJ, 2018.

[56] GOMIDE, V. S., "Desenvolvimento e caracterização mecânica de compósitos hidroxiapatita-zircônia, hidroxiapatita-alumina e hidroxiapatita-titânia para fins biomédicos", Tese de D.Sc., Unicamp, Campinas SP, 2005.

\section{ORCID}

Jailton Romão Viana

Ana Angélica Mathias Macêdo

Adenilson Oliveira dos Santos

Pedro de Freitas Façanha Filho

Manuel Pedro Fernandes Graça

Cleber Cândido da Silva https://orcid.org/0000-0002-8790-0217

https://orcid.org/0000-0003-4750-2721

https://orcid.org/0000-0002-2248-5104

https://orcid.org/0000-0003-4979-7287

https://orcid.org/0000-0002-6858-9507

https://orcid.org/0000-0002-2698-4138 